\title{
Step-On Interface on Mobile Robot to Operate by Stepping on Projected Button
}

\author{
Takafumi Matsumaru* and Kosuke Akai
}

Bio-Robotics and Human-Mechatronics Laboratory, Shizuoka University, Johoku, Naka, Hamamatsu 432-8561, Japan

\begin{abstract}
This paper proposes the step-on interface (SOI) to operate a mobile robot in which a projector projects and displays a direction screen on a floor and a user specifies a button showing the selected movement by stepping or pointing. The HFAMRO-1 mobile robot has been developed to demonstrate the SOI's potential (HFAMRO: "human-friendly amusing" mobile robot). The SOI of HFAMRO-1 consists of a projector and a range scanner on an omni-directional mobile platform. From operational comparison with other input interfaces, it is confirmed that we can direct the robot movement using our own foot. We had some students who does not specialize robotic systems try to operate HFAMRO-1 with their shoes, and all trial students could specify the button to operate the robot satisfactorily and everyone mastered the SOI immediately.
\end{abstract}

Keywords: Step-On Interface (SOI), Human-Friendly Amusing Mobile Robot (HFAMRO), Projector, Range scanner, Direction screen.

\section{INTRODUCTION}

This paper proposes the step-on interface (SOI) to operate a mobile robot in which a projector projects and displays a direction screen on a floor and a user specifies a button showing the selected movement by stepping or pointing. The HFAMRO-1 mobile robot has been developed to demonstrate the SOI's potential (HFAMRO: "human-friendly amusing" mobile robot) [1-4]. The SOI of HFAMRO-1 consists of a projector and a range scanner on an omnidirectional mobile platform.

This paper is organized as follows: Section 2 explains the feature of the SOI comparing with related studies and conventional methods. Section 3 introduces the HFAMRO-1 mobile robot. Section 4 and 5 explain the measurement method and the direction procedure when the robot is idle and when it is operating respectively. Section 6 shows setup movements of HFAMRO-1. Section 7 discusses experiments. Section 8 presents conclusions.

\section{STEP-ON INTERFACE}

\subsection{Conventional Interface Using Projector}

Human interface (HI) means, in a narrow meaning, a connection part between a person and a machine or a computer, and is an equipment to make user easy to operate a machine [5]. Interface usually informs multimedia information and a display presents information visually via a screen such as CRT (cathode-ray tube), LCD (liquid crystal display) and projector (projection equipment).

Digital Desk [6] proposed by Wellner in 1991 has changed previous indirect relationship between a user and a

*Address correspondence to this author at the Bio-Robotics and HumanMechatronics Lab., Shizuoka Univ., Johoku, Naka, Hamamatsu 432-8561, Japan; E-mail: ttmatum@ipc.shizuoka.ac.jp computer using a screen and a computer mouse. On Digital Desk, three functions are considered: visually representation function (a visual desktop is projected on a desk), control function (user's own fingertip is utilized to direct operation), and registration function (objects such as documents and figures are input electronically in the desktop environment as information resources using, for example, a camera). These three functions are studied as Enhanced Desk [7], Sensetable [8], Illuminating Clay [9], SmartSkin [10], Lumisight Table [11], and Attentive Workbench [12] and some prototype projects vertically on a wall $[13,14]$. These three functions are visual interaction, however, a reaction function is studied so that a reaction force against operation is fed back to a user such as PSyBench (physically synchronized bench) [15], Visual Haptic Workbench [16], Actuated Workbench [17], and Proactive Desk [18]. However all these interactions are only between a user and a virtual environment.

Projector is sometimes used to support handling a real object, for example, it displays information from a remote supporter to an on-site worker to support and prescribe tasks or operations [19, 20]. RFIG (radio frequency identification and geometry) lamp (local-aware mobile projector) [21] is interesting as an informational interaction in real workspace which uses an active RFID tag with photo electronic sensor and a handheld projector with camera. ID information and location of tags in readable area are recognized using a gray code light pattern from the projector, and the information is projected on each object. As an interface between a user and a robot, projector is usually used for teaching [22-24], but it is mainly off-line and the operation and direction of working robot are seldom examined. The information sharing function on manipulator [25] and the preliminary-announcement and indication function of upcoming operation on mobile robot [26] are studied to present user a robot's movement information via projector, however, it transmits information only from robot to user. 


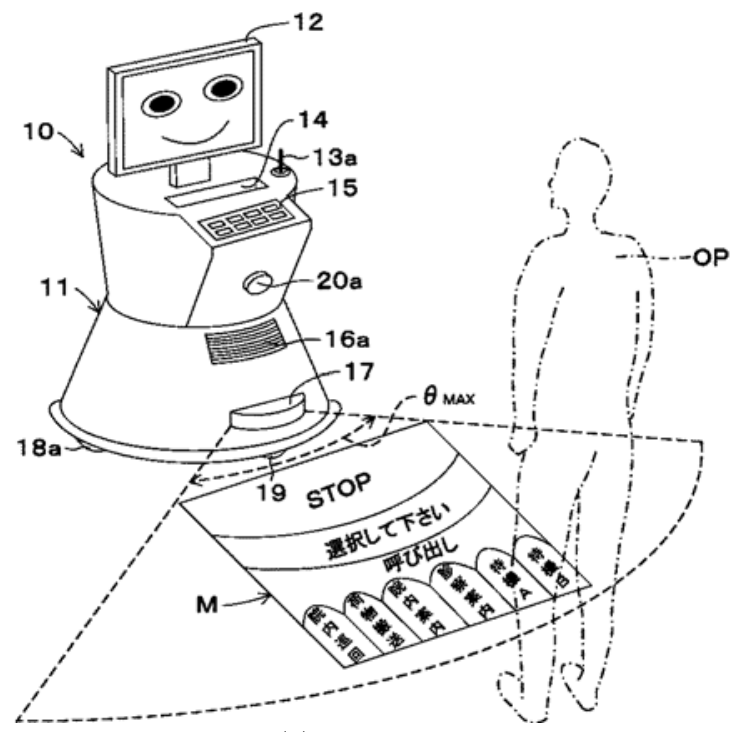

(a) Projection

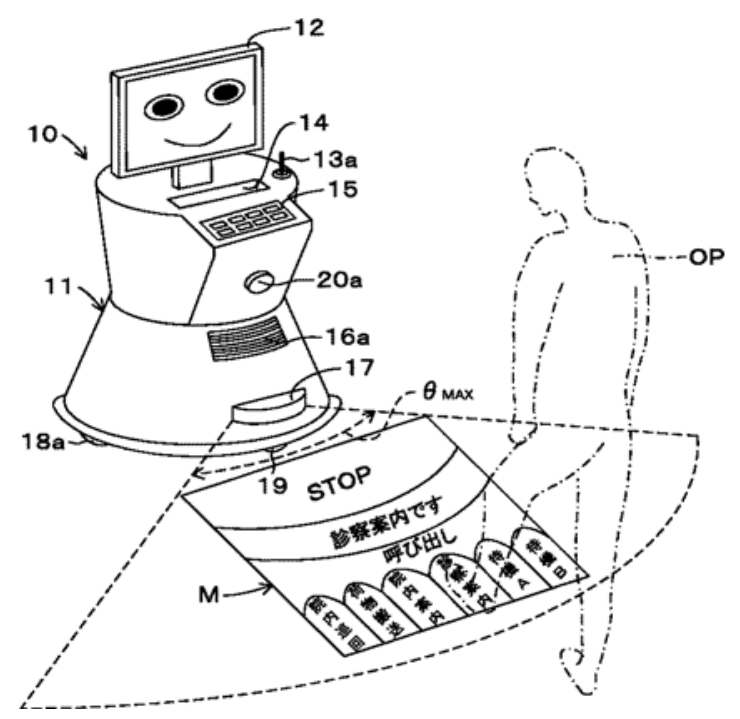

(b) Stepping-on

Fig. (1). SOI on In-Hospital Service Robot.

\subsection{Step-On Interface}

The SOI is a new interface to direct robotic or mechartonic tasks or operations. A projector displays a direction screen on a floor or other surface, and a user specifies a button showing the selected movement by stepping or pointing. The SOI is considered, for example, to be equipped on a support robot in hospital or nursing facility (Fig. 1) [27]. In many cases still more supports with one more pair of hands are needed to assist those whose busy hands are already full - nurses, doctors, and care workers in the quickly aging society. Accordingly a human coexisting type robot which performs, for example, delivery or conveyance of medical appliances, patient's records and meals is desired.

The SOI has a special feature that the projected screen is used as a bilateral interface. It not only presents information from equipment to user but also delivers instructions from user to equipment. The SOI has the following features:

(1) The SOI can be used hands-free, unlike keyboard and computer mouse, touch panels, and joysticks. Therefore the elderly, the physically challenged, and users whose hands are full can use it.

(2) Because the SOI does not need voice input, it can be used anywhere without disturbing others and in noisy environments.

(3) No special devices are needed, and the user can use his own foot or stick (walking case) usually used, although the eye control requires special devices to detect eye movement and has constraints such as user's position and orientation to be recognized.

(4) A user only moves his foot or stick roughly to direct, instead of hands and fingers movement with high accuracy.

(5) Direction screen projection means that the SOI requires little preliminary preparation or special setup.

(6) Because the SOI can use figures and pictures in addition to letters, it is no specifically language dependent, making it possible for beginners and non-native speakers to use it.

(7) Screen functions are easy to design, setup and change because they are included in software.

\subsection{Comparison with Conventional Method}

Conventional input by users may be manual operation, voice input and eye control (Table 1). Manual operation uses push-pull button, joystick, keyboard and computer mouse, touch panel (touch screen) [28, 29], which are not always possible for the elderly or physically challenged. Voice input

Table 1. Comparison Among Various Input Devices

\begin{tabular}{|c|c|c|c|c|}
\hline & Manual & Voice & Eye control & SOI \\
\hline Hands-free & - & $\mathrm{AA}$ & $\mathrm{AA}$ & $\mathrm{AA}$ \\
\hline Skill to use & AA & A & - & AAA \\
\hline Environmental condition & AA & - (noisy) & A (lighting) & A (lighting) \\
\hline Operating efficiency & AAA & - & - & AAA \\
\hline
\end{tabular}




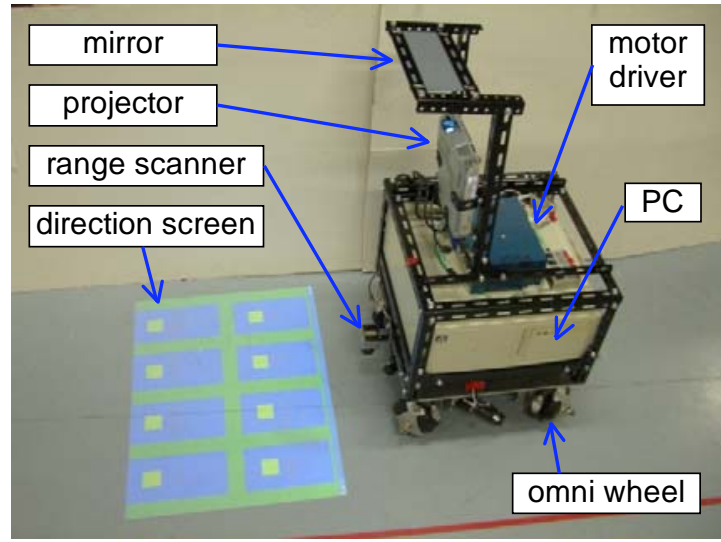

Fig. (2). HFAMRO-1.

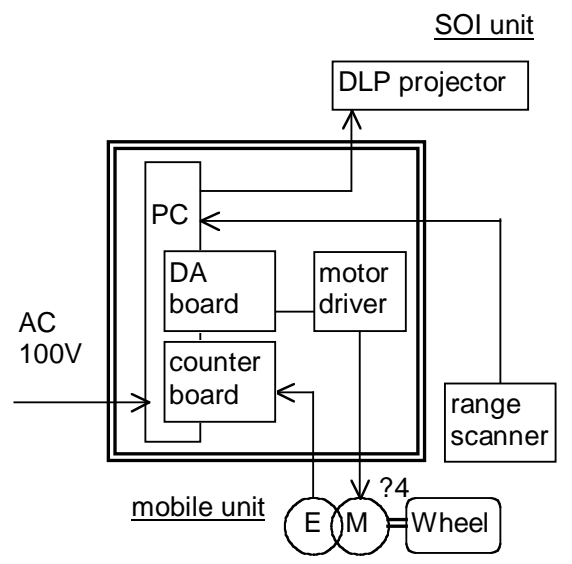

Fig. (3). HFAMRO-1 system configuration.

Table 2. HFAMRO-1 Specifications

\begin{tabular}{|c|c|}
\hline Item & Specification \\
\hline \hline Size & $\mathrm{D} 450 \times \mathrm{W} 450 \times \mathrm{H} 960 \mathrm{~mm}$ \\
\hline Weight & $46.7 \mathrm{~kg}$ \\
\hline Max. speed & $220 \mathrm{~mm} / \mathrm{s}, 0.87 \mathrm{rad} / \mathrm{s}$ \\
\hline
\end{tabular}

$[30,31]$ has difficulties in recognition, sometimes due to direction, noise, speech peculiarities, and language translation. Also it occurs "noise" to disturb quietness and give unpleasant to others, and it cannot keep communicated content from being understood by others. Eye control $[32,33]$ requires neither special instruments nor the hands for users to input command but does require practice, mastering, and familiarity. Limitations may exist such as lighting conditions or user's position and orientation to be recognized.

"Small Fish" [34] is a representative example of media artwork featuring user-equipment interaction using projector. The symbols (the translucence or real objects with round or rectangle shape, the cushions of a doughnut form, a log form, an asteroid form, etc.) handled by users on a projected screen are detected by an infrared camera and a motiontracking software, and the system provides sound and changes contents based on object conditions and user opera- tions. "Tap Talk" [35], a commercialized example of userequipment interaction acts as an interactive floor screen on which an image reacts and changes when people walk over an image projected on the floor. The motion of people and other materials is detected by an infrared camera. The system limitation lies in the fact that the camera and projector are assumed to be fixed to the ceiling, the floor image location is fixed beforehand, and the object handled is not actual object but is multimedia information. It also does not consider the application as an interface for artificial mechatronic systems. A virtual keyboard (projection keyboard) [36], similar to the SOI and marketed as computer peripheral equipment, projects an outline of a keyboard on a desk using a red semiconductor laser. A built-in infrared sensor detects the keys "touched". This equipment assumes a precise professionaltypist key touch, so the elderly or physically challenged may not use it easily.

\section{MOBILE ROBOT EQUIPPED WITH SOI}

\subsection{HFAMRO-1 Mobile Robot}

The HFAMRO-1 mobile robot, developed to demonstrate the SOI's potential, uses a SOI consisting of a projector and a range scanner on an omni-directional mobile platform. The projector displays a direction screen on a travel surface and the two-dimensional (2D) range scanner which scans on a plane and measures the distance to an object detects and measures the user's stepping to specify the selected button on the direction screen. HFAMRO is intended represent the concept based on which robots interact with users with its mobility, for example, the ability to play tag - in this case playing tag with "light", similar to "shadow" tag. This assumes a scenario in which a child runs after a mobile robot and, when a button on the projected screen is stepped on, the robot responds, for example, by playing a game like rockpaper-scissors or a riddle, giving the time or weather report, and moving in some way to indicate an emotion.

\subsection{System Configuration}

Outward appearance, system configuration and principal specifications of HFAMRO-1 are shown in Figs. $(\mathbf{2}, \mathbf{3})$, and Table 2 respectively. The omni-directional mobile platform consists of four sets of omni-wheels driven by a DC motor, reduction gears, and an encoder. It can move in any direction keeping its orientation and also rotate centering on an arbitrary point on a travel surface.

The SOI consists of the display in the form of a projector and the detector in the form of a range scanner. A commercial product, V3-131 by Plus Vision Co., used as the projector, faces upward and the screen is reflected in the mirror above it and projected on a travel surface. This keeps the robot height under $1 \mathrm{~m}$ while minimizing projection distance to $1.2 \mathrm{~m}$. The mirror is installed so as to be inside the robot's main body ( $450 \mathrm{~mm}$ square) to reduce the risk to contact with users or objects around. The mirror is installed with inclination angle of 5 deg so that the robot's main body does not interfere with screen projected. The trapezoid compensation makes the projected screen a rectangle $(730 \mathrm{~mm}$ wide and $550 \mathrm{~mm}$ long) on a travel surface. A commercial product, URG-X002S by Hokuyo Automatic Co. [37], used as the range scanner, scans 682 steps across 240 deg (step number: 1 to 682 ; step angle: $0.35 \mathrm{deg}$ ), determining the dis- 


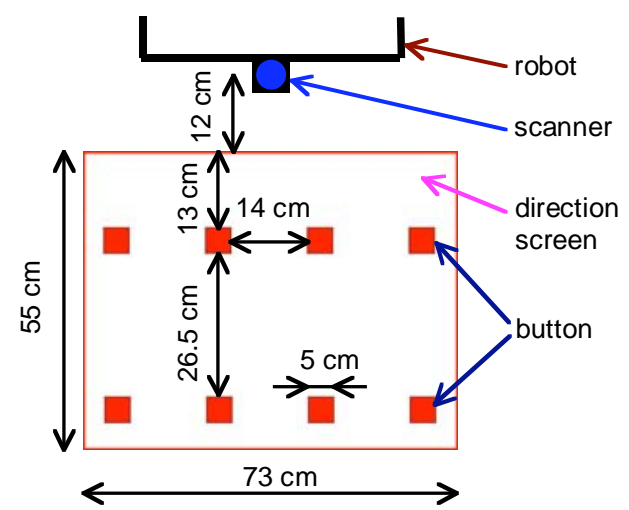

Fig. (4). Placement of buttons in direction screen.

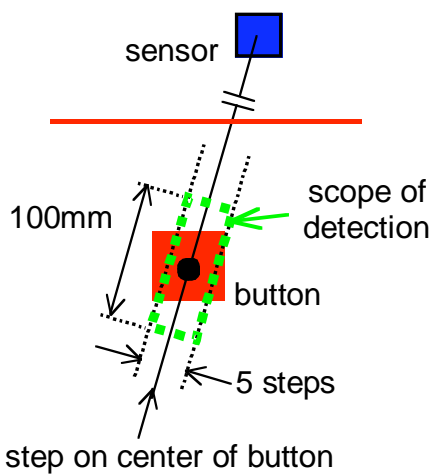

Fig. (5). Scope of detection when robot is idle.

tance from 20 to $4095 \mathrm{~mm}$ to an obstacle via RS-232C or USB. Located on lower front center of the robot, the range scanner is roughly $20 \mathrm{~mm}$ above the travel surface keeping from touch with the surface unevenness.

\subsection{Button Arrangement in Direction Screen}

The 50-mm-square buttons are placed in two lines by four sequences on the direction screen $(730 \mathrm{~mm}$ wide and $550 \mathrm{~mm}$ long) (Fig. 4). It is based on the range scanner performance in experiments [38] and the investigation reports on foot length $(21.1 \mathrm{~cm}$ to $28.7 \mathrm{~cm})$ and footwear size (25EE) of common male adults (18 to 60 years old) in Japan [39].

\section{SOI OPERATION WHEN ROBOT IS IDLE}

The direction screen when the robot is idle is designed considering that we can select and direct as many operations as possible from a screen in which buttons are placed in two lines by four sequences.

\subsection{Recognition Method and Judgment of Stepping}

The robot can acquire the step number (direction angle) and the distance value to an object as position data from the range scanner, and the existence of foot or footwear on a operation button is judged using the distance values at adjacent five steps (equivalent to $1.4 \mathrm{deg}$ and, for example, 16 $\mathrm{mm}$ wide at the distance of $65 \mathrm{~cm}$ from the scanner) including each two steps right and left with the center step passing through the button center. It is because a misjudgment due to measurement error of range scanner may occur if checking the distance only at the step corresponding to the button center. We consider the operation button size (50-mm-square), the maximum measurement error of range scanner (about 20 $\mathrm{mm}$ ), and the individual difference by users on the buttonfoot relative position from a pilot study [38]. Consequently the scope of detection is set at the distance from the scanner to the button center plus or minus $50 \mathrm{~mm}$ (Fig. 5). When the distance at all five steps is in the scope of detection, it is judged that a user steps on the button to direct the operation indicated.

\subsection{Hierarchy}

The hierarchical structure is introduced since the number of button which can be placed on a direction screen is limited to eight, two lines by four sequences, and the number of operation directed from a screen is restricted to eight. The operations selected are detailed from robot side to user side on the screen. That is, when one of four classification buttons placed in the upper line is stepped (Fig. 6(a)), four individual buttons which show lower-rank operations detailed under the classification are displayed in the lower line (Fig. 6(b)). By this hierarchy, sixteen kinds of operation, four classifications by four individuals, can be selected and directed when the robot is idle.

\subsection{Direction Procedure}

The direction procedure from button selection to robot movement is as follows (Fig. 7):

(1) The projector projects the direction screen on a floor (Fig. 8(a)).

(2) The range scanner scans on the screen and it acquires the position data (angle and distance).

(3) When all the distances checked at five steps are in the scope of detection, it is judged that the button is

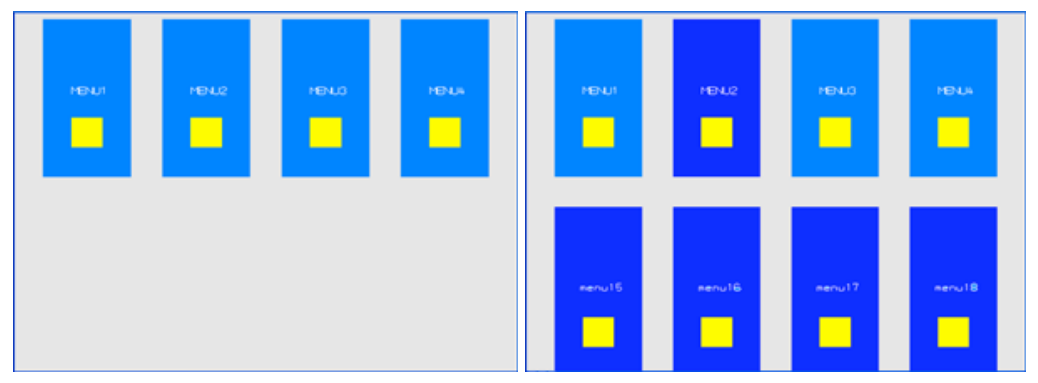
(a) 1st stage
(2) 2nd stage

Fig. (6). Hierarchical structure for direction screen. 


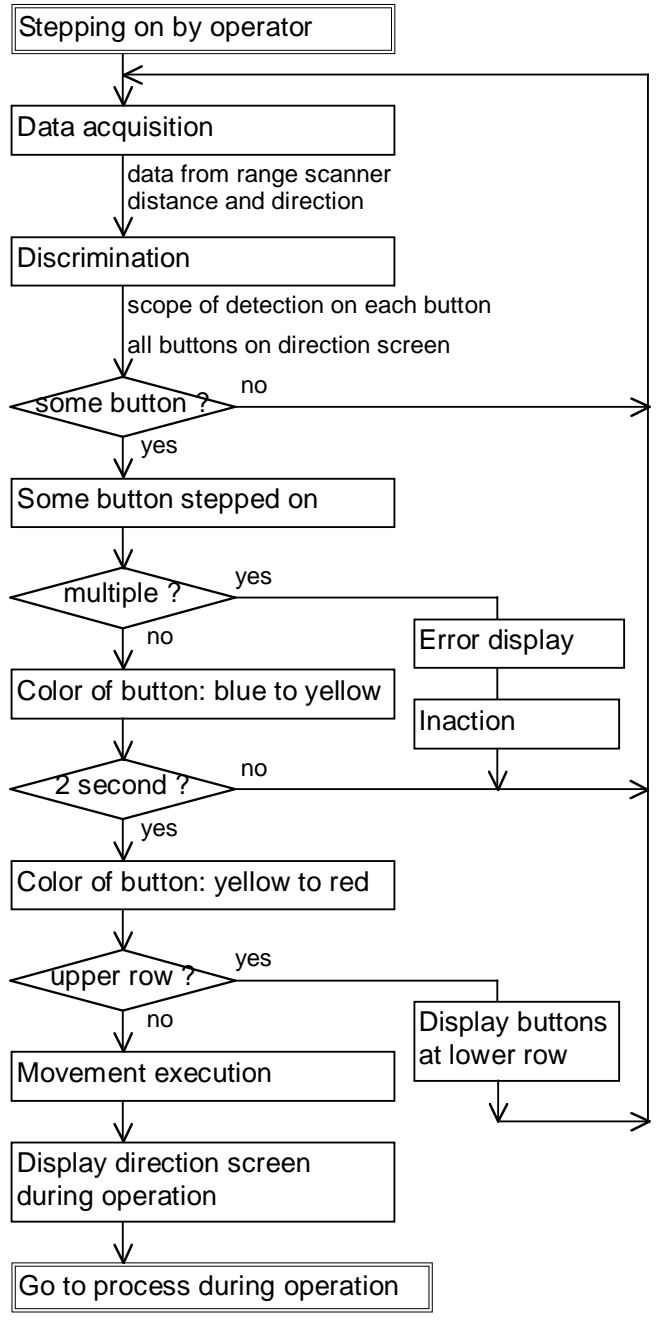

Fig. (7). Direction procedure when robot is idle.

stepped. The system doesn't have any sensory feedback corresponding to keyboard typing feeling, so the color inside the frame around the button (i.e. button frame) is changed from usual blue to yellow to inform the user that the system recognizes the button stepped (Fig. 8(b)).

(4) The system doesn't immediately perform the operation considering that the user makes a mistake in stepping or the system fails to recognize. When the system recognizes the button stepped for two seconds (the period can be easily changed in software), the color inside the button frame is changed from yellow to red and the operation is performed (Fig. 8(c)).

(5) While two or more buttons stepped are recognized simultaneously, any direction is ignored and the error indication is displayed on the screen (Fig. 8(d)). When the error is resolved, the direction screen goes back to the previous state.

\section{SOI OPERATION WHEN ROBOT IS OPERATING}

When the robot comes into operation responding to the user's direction, the direction screen is transformed. The judgment of stepping is designed considering that operations can be performed without interruption.

\subsection{Recognition Method}

When the robot is operating, the direction screen moves from the user with the robot movement, conversely from the range scanner, the user's stepping location moves. So we have to make the scope of detection for each button wider, and we made the scope of detection for each button all areas inside the button frame.

As stated, when the robot is idle, a part of ring centering on the range scanner is set as the scope of detection to judge the button directed by user, so the position data (step number and distance) acquired from range scanner can be used directly. However, when the robot is operating, if applying this, we have to make the part of ring wider than the button frame. This makes it difficult to correctly detect the button stepped because the scope contains other button frames and overlaps each other (Fig. 9).

Accordingly the position data acquired from range scanner is converted to X-Y Cartesian coordinates, and it is investigated whether the $X-Y$ value is in the scope of detection corresponding to the button frame. When there are five or more $\mathrm{X}-\mathrm{Y}$ values in the scope of detection, it is judged that a user directs the operation indicated on the button (Fig. 10).

\subsection{Judgment of Stepping}

A new operation is performed immediately when the system recognizes a button stepped when the robot is operating. Even though the scope of detection is set widely as all inside the button frame, the same state is not necessarily kept for a period because the robot is moving. For example, if the user's foot stepped on a button stays in the same place, the button judged as directed changes one after another with the robot movement. Therefore, when the robot is operating, next direction is ignored for one second (the period can be easily changed in software). Nevertheless the 'stop' button is always given top priority to make the robot stop immediately. In addition each operation has a time limit so that after some predefined period the robot stops and the direction screen goes back to select individual buttons. Even if two or more buttons stepped are recognized simultaneously, the robot continues the current operation because the interruption reduces the robot maneuverability for users.

On the direction screen, in principle, the large 'stop' button is set in the upper line and other operation buttons are placed in the lower line (Fig. 11). The operation buttons should be placed in a line because, if the operation buttons are arranged in two lines, the operation button in lower line could incorrectly react in response to the user's foot returning from stepping an operation button in the upper line. It is also possible when a user returns his foot after stepping the 'stop' button in the upper line, the operation button in the lower line is misrecognized as stepped and the robot executes the next operation. So, when the stop button is recognized, the recognition of other buttons is ignored for two seconds (the period can be easily changed in software). 


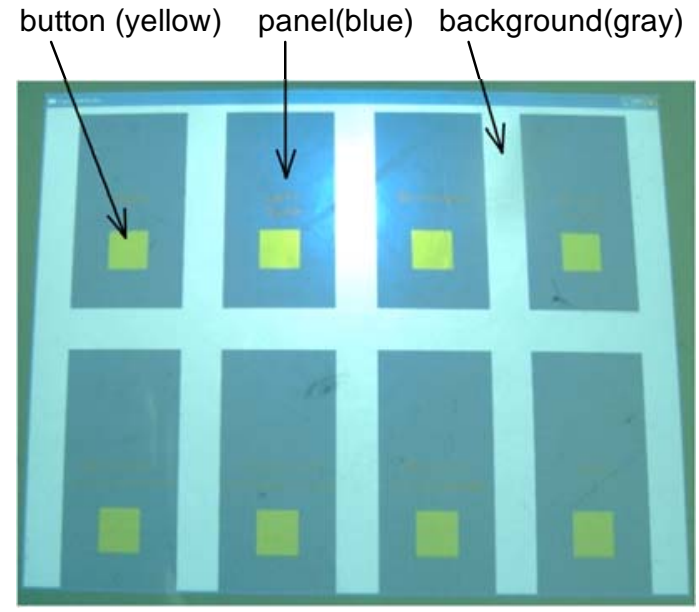

(a) Readiness

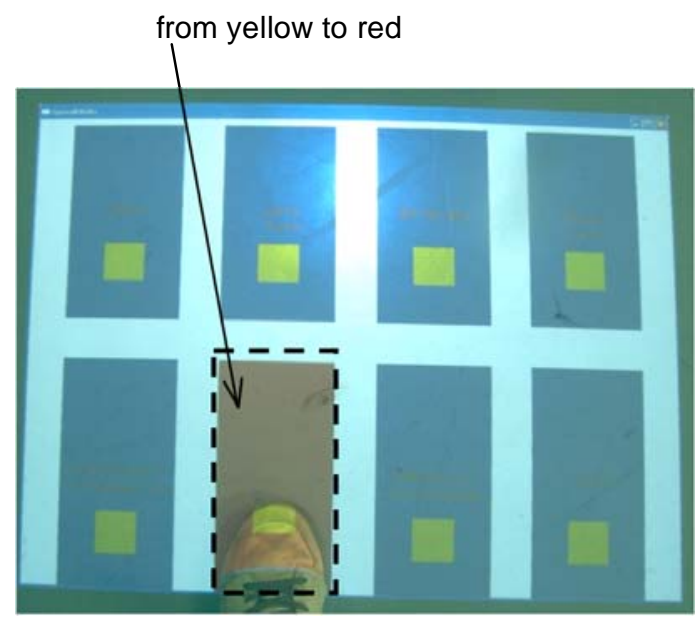

(c) Execution

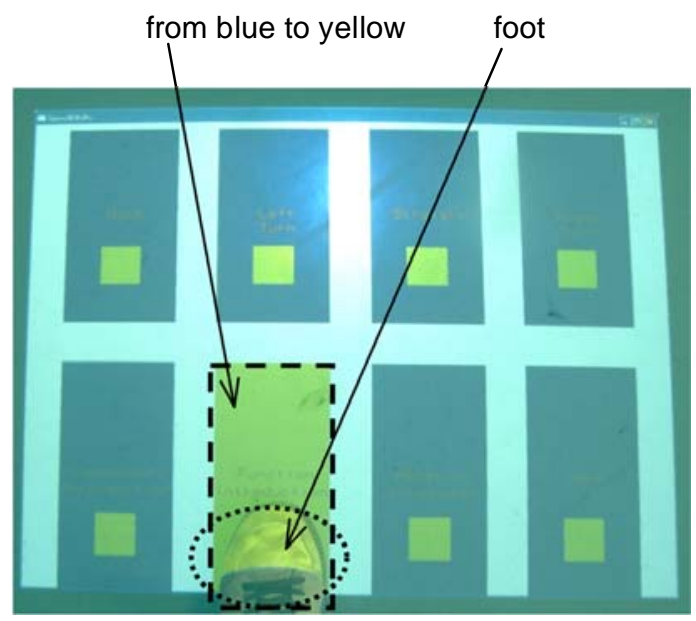

(b) Detection

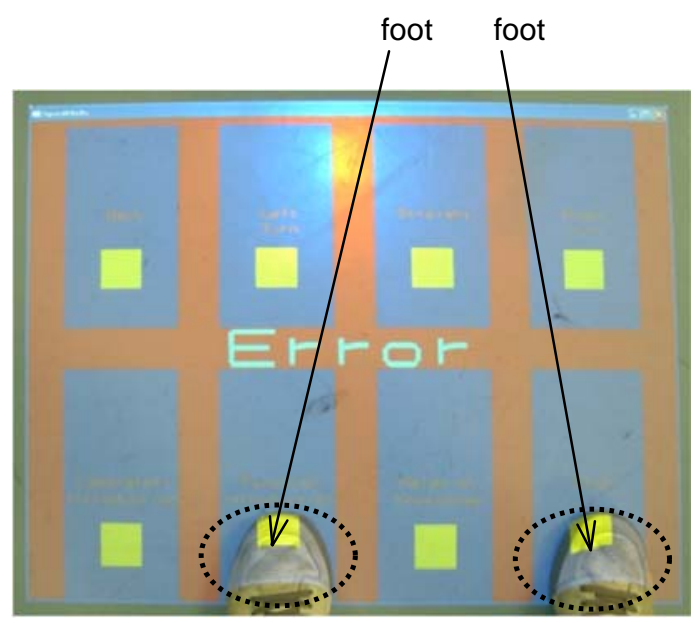

(d) Error

Fig. (8). Direction screen when robot is idle.

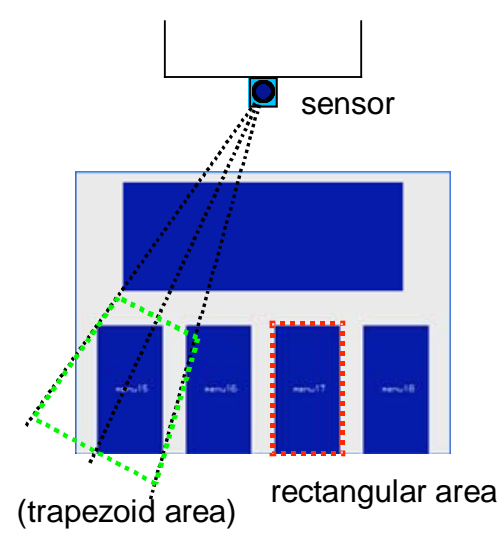

Fig. (9). Scope of detection when robot is operating.

\subsection{Direction Procedure}

The direction procedure from button selection to robot movement is as follows (Fig. 12):

(1) The projector projects the direction screen on a floor.

(2) The range scanner scans on the screen and it acquires the position data (angle and distance).

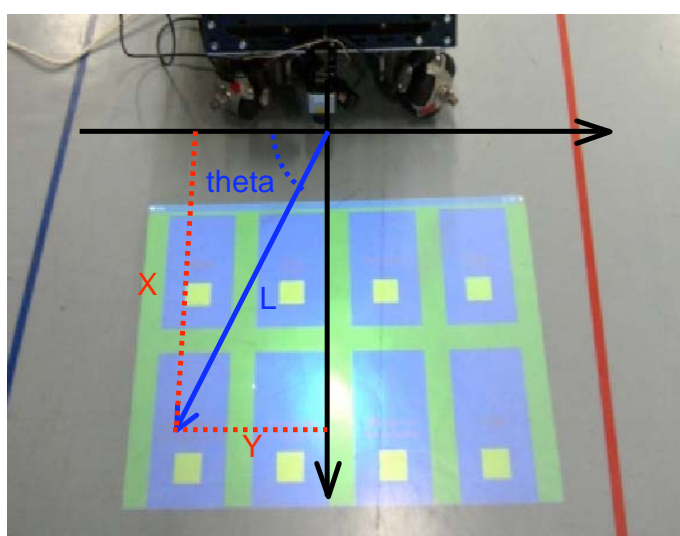

Fig. (10). Coordinate conversion.

(3) The acquired position data is converted to X-Y Cartesian coordinates.

(4) When five or more X-Y values are there inside the button frame, it is judged that the button is stepped.

(5) When the system recognizes a button stepped, it informs the user by changing the color inside the button 


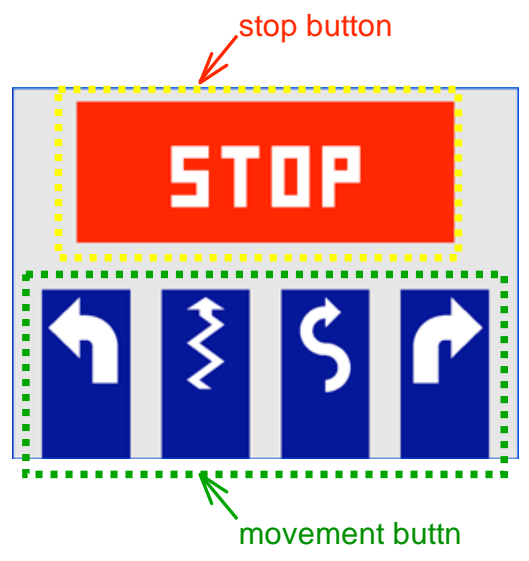

Fig. (11). Direction screen when robot is operating.

frame from usual blue to red and the robot performs the operation immediately.

(6) While two or more buttons stepped are recognized simultaneously, the robot continues the current operation.

(7) After some predefined period the robot stops and the direction screen goes back to select individual buttons.

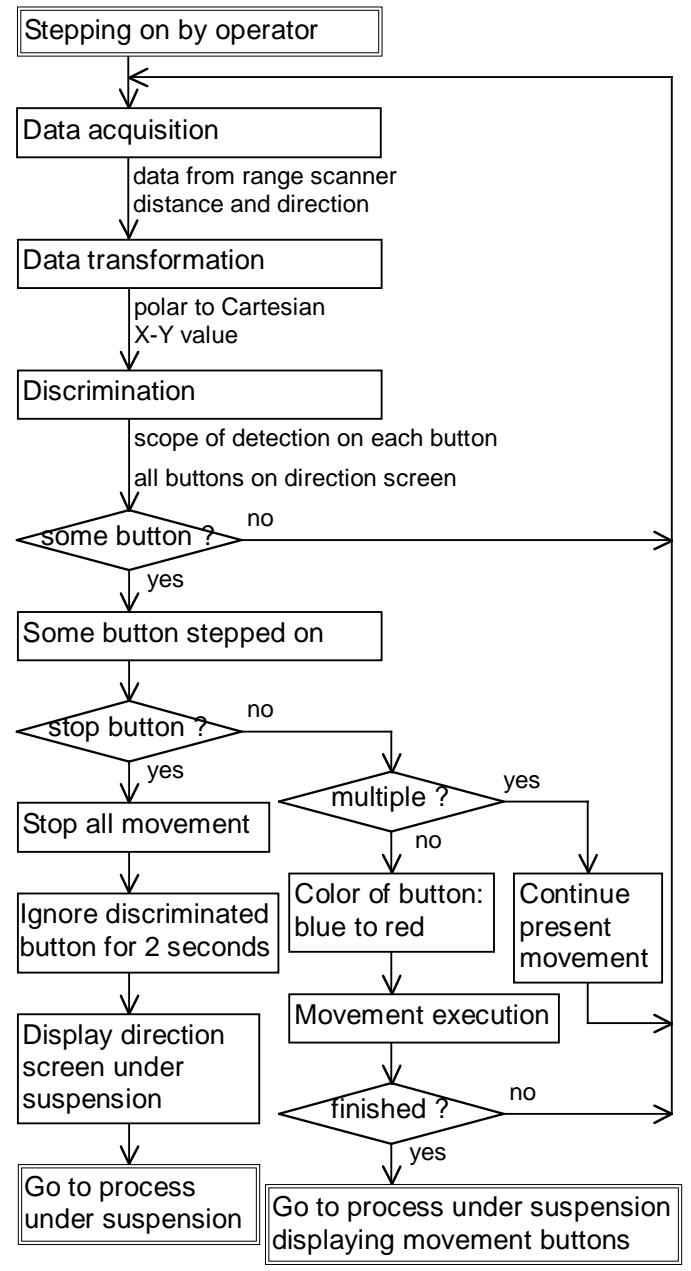

Fig. (12). Direction procedure when robot is operating.

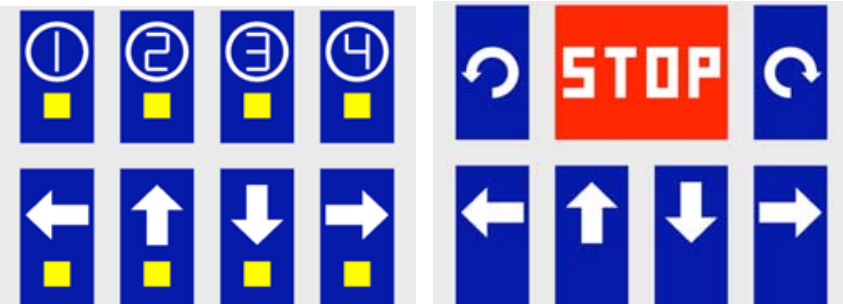

(a) When robot is idle

Fig. (13). Direction screen for basic movement.

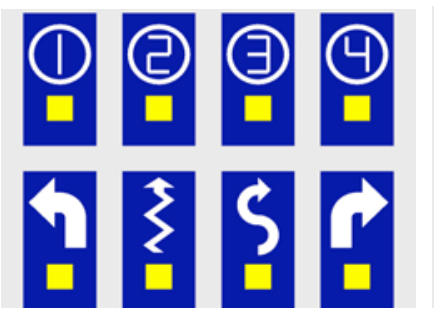

(a) When robot is idle

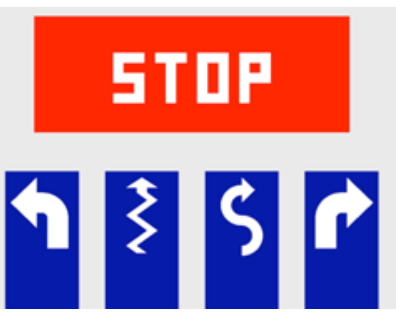

(b) When robot is operating

Fig. (14). Direction screen for elemental movement (1st half).

\section{SETUP MOVEMENT OF HFAMRO-1}

In HFAMRO-1 eighteen kinds of movement are prepared: six kinds of basic movement, eight kinds of elemental movement, and four kinds of emotional movement.

\subsection{Basic Movement}

Basic movement means a fundamental motion. Six kinds of movement can be considered in a omni-directional mobile platform: back-and-forth translations, right-and-left translations, and clockwise-and-counterclockwise rotations. On the direction screen when the robot is idle, only four kinds of translation are displayed as individual button (Fig. 13(a)). On the direction screen when the robot is operating, the operation buttons are set not only in the lower line but also at right and left in the upper line (Fig. 13(b)) since we want to select all the six basic movements on the direction screen. Even if a user keeps his foot stepped on the upper right or left buttons, the system may not operate incorrectly because these two buttons are assigned to rotation and the direction screen leaves from the user when the robot starts operations. The 'stop' button is placed at the upper center so the operator's stepping never contact with robot's body. Basic movements can be selected on the direction screen from the classification button-1 when the robot is idle.

\subsection{Elemental Movement}

Elemental movement is a complicated motion combining basic movements and other movements based on trigonometric functions, etc. Operations called 'left turn', 'zigzag', 'slalom', and 'right turn' can be selected on the first direction screen displayed from the classification button-2 (Fig. 14), and operations called 'circle', 'step turn', 'box', and 'diagram' can be selected on the second screen from the classification button-3 (Fig. 15). Buttons for elemental movements are shown with icons indicating robot's trajectory. 


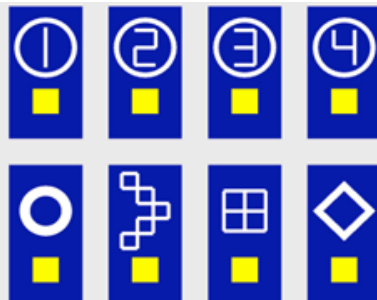

(a) When robot is idle

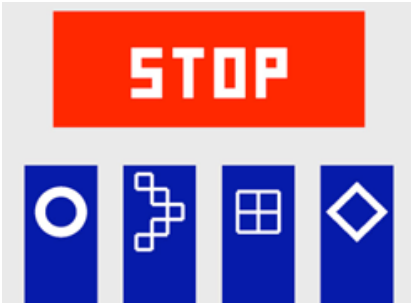

(b) When robot is operating

Fig. (15). Direction screen for elemental movement (2nd half).

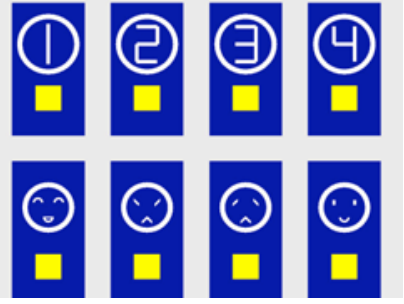

(a) When robot is idle (b) When robot is operating

Fig. (16). Direction screen for emotional movement.

\subsection{Emotional Movement}

Emotional movement is designed aiming to express robot's 'emotion' only with its movement. Four kinds of movement - 'joy (pleasure)', 'anger (spoiled child)', 'sadness (depression)', and 'happiness (grace)' - are set on trial (Fig. 16). It can be selected on the direction screen from the classification button- 4 when the robot is idle (detailed explanation about movements is omitted because of space limitation). Buttons for emotional movements are presented with moonfaced icons with eyes and mouth.

\section{EXPERIMENTAL EVALUATION}

\subsection{Operational Comparison with Other Input Interfaces}

The feature of SOI is studied based on the HFAMRO-1 operations with keyboard, voice, and SOI.

We asked a subject, undergraduate student who is not expert at robotic system, to move the robot along a trajectory drawn on the floor with straight lines $1 \mathrm{~m}$ long and the rotation angle of $45 \mathrm{deg}$ (Fig. 17). The robot is set to move at a speed of $120 \mathrm{~mm} / \mathrm{s}$ (translation) and $0.34 \mathrm{rad} / \mathrm{s}$ (rotation),

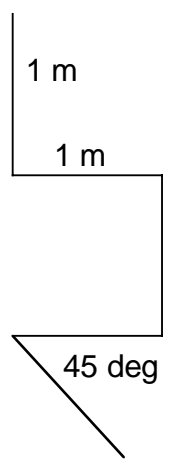

Fig. (17). Desired trajectory.

and the subject directs only the movement direction and the temporary stopping. Reverse run is prohibited even if the robot overruns. A 10-key part of 106-keyboard [40] as a keyboard, the Julius for SAPI (speech application program interface) [41] as a speech recognition system for voice input, the direction screen for basic movements for the SOI (Fig. 13) are used respectively. Setup is shown in Table 3.

The movement loci are shown in (Fig. 18). By keyboard (required time: $48 \mathrm{~s}$, rotation angle: $45.2 \mathrm{deg}$ ), the difference between the target and the result is the smallest. It shows if a user can use his hands and fingers satisfactorily he can operate the robot precisely. By voice (required time: $49 \mathrm{~s}$, rotation angle: $49.7 \mathrm{deg}$ ), the difference is the largest - more than $10 \mathrm{~cm}$ at the maximum. The movement changeover at aimed direction in rotation is especially difficult due to the time lag in the speech recognition system. By SOI (required time: $47 \mathrm{~s}$, rotation angle: $48.5 \mathrm{deg}$ ), the difference is not so large, but the movement changeover is comparatively difficult because the direction screen moves with the robot movement. Nevertheless it is confirmed that we can operate the robot with our own foot using SOI if the task dose not require high accuracy.

\subsection{Trial Operation of HFAMRO-1}

We brought the HFAMRO-1 to a campus cafeteria and had some students, who do not specialize robotic systems, try to operate HFAMRO-1 with their shoes (Fig. 19). Fifteen students replied to the questionnaire after the trial.

Table 3. Setup of Input Interface

\begin{tabular}{|c|c|c|c|}
\hline \multirow{2}{*}{ Robot Movement } & \multicolumn{3}{|c|}{ Input Device } \\
\hline & Keyboard & Voice & SOI \\
\hline Going forward & '8' key & "mae" = fore & button with symbolic icon \\
\hline Going backward & '2’ key & "ushiro" = back & button with symbolic icon \\
\hline Going leftward & ‘4’ key & "hidari" = left & button with symbolic icon \\
\hline Going rightward & ‘6’ key & "migi" = right & button with symbolic icon \\
\hline Left rotation & '7' key & "kaiten" = rotate & button with symbolic icon \\
\hline Stop & '5’ key & "teishi" = stop & button with characters \\
\hline
\end{tabular}




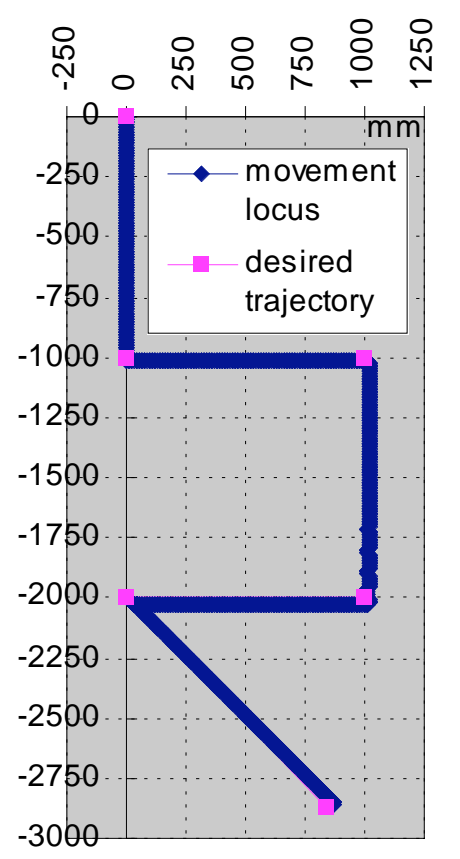

(a) Keyboard

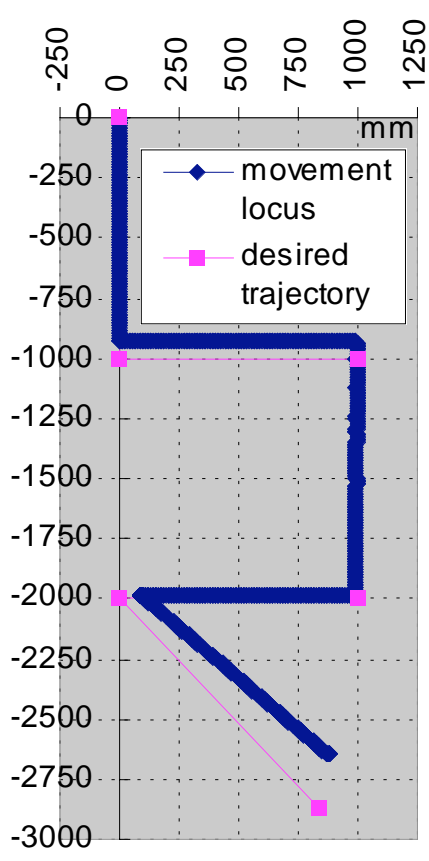

(b) Voice

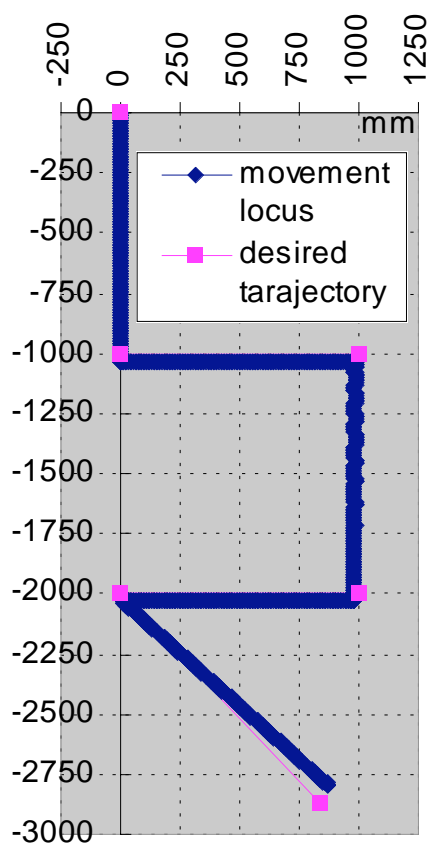

(c) SOI

Fig. (18). Movement locus of HFAMRO-1.

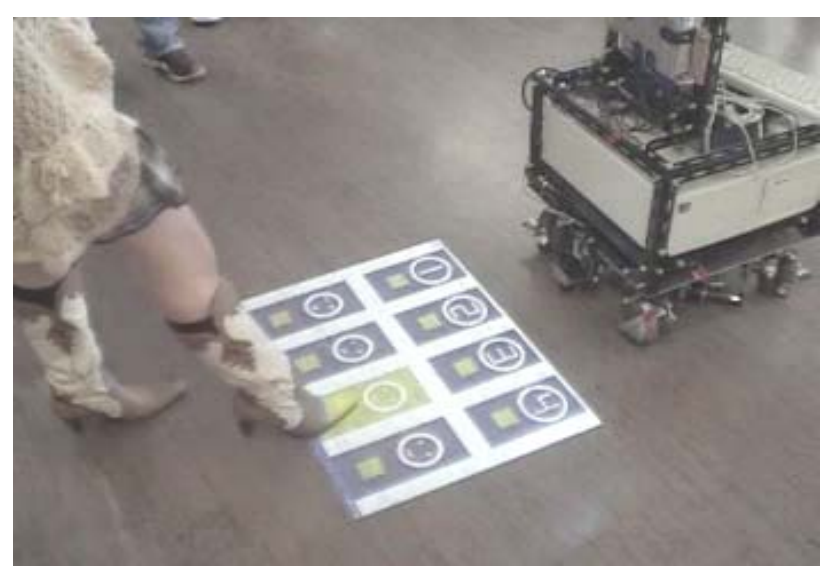

Fig. (19). Trail operation of HFAMRO-1.

Table 4. Robot Maneuverability

\begin{tabular}{|c|c|}
\hline Maneuverability & Number of People \\
\hline \hline Easy to operate & 10 \\
\hline Reasonable to operate & 3 \\
\hline Difficult to operate & 0 \\
\hline (No opinion) & 2 \\
\hline Total & 15 \\
\hline
\end{tabular}

\subsubsection{Questionnaire Result}

The subjects noted the kind of shoes they worn and answered the threefold choice about robot maneuverability - easy to operate, reasonable to operate, and difficult to operate (Table 4). No one answered difficult to operate.

- Shoes of ten students answered easy to operate: white sneaker (3), black sneaker (3), silver sneaker (1), dark blue with gray sneaker (1), black mesh material (1), black leather shoes (1).

- Shoes of three students answered reasonable to operate: brown sneaker (2), beige leather boots (1).

All trial students including those who did not reply to the questionnaire could specify the button to operate the robot satisfactorily and everyone mastered the SOI immediately beyond our expectation. Many students looked for the location at which the SOI responds moving around their toe at first because the portion of shoes which the range scanner detects is depending on shoes color, size, and shape. A student who worn black shoes presented tips as raising heel and tiptoeing. The SOI seems to be less responsive to the upper right and left buttons on the direction screen because the shoes facing to the robot at the upper corner on the direction screen have some inclination angle to the scanner and the amount of reflected light to the scanner may be reduced. Accordingly we should examine the recognition and judgment of shoes with various materials and shapes, and exercise the ingenuity on shoes orientation such as the design and placement of buttons to let the user turn his shoes facing to the scanner.

\subsubsection{Comments and Opinions}

Comments and opinions filled in the free entry are considered as follows:

\section{ADVANTAGE AND DISADVANTAGE}

Many students understand the advantage of SOI mentioning that, for example, anyone can operate the robot without 
his hands even if he carries some loads or baggage. Disadvantage is also pointed out such that the direction screen moves with the robot movement and a user has to run after the robot to direct it sequentially. This might be a serious problem depending on its application and situation. Some student requests the function to display the direction screen around the user continuously as in remote operation. The interruption function after it leaves somewhat away from the user is a simple and immediate countermeasure.

\section{USAGE ENVIRONMENT}

It is also pointed out that a user is difficult to recognize buttons when a floor has some unevenness or clear pattern, and that it is dangerous if others step on undesired buttons, e.g., in a crowd. The system is assumed to be used indoors at first. As for the detection and measurement of user's stepping, we have to examine, for example, the recognition and judgment of shoes processing shape data acquired from range scanner or usage of other sensors to distinguish the user from others and obstacles. We plan to use the SOI on various applications examining its implementations.

\section{EMOTIONAL MOVEMENT}

Many positive comments are there on emotional movement, especially on the anger expression - swaying its body like a child throwing a tantrum. However, there are also negative opinions such that it is difficult to understand without explanation and there is not much difference among four emotional movements. We would like to examine more on various expressions with improving robot mobility. We would like to apply the SOI not only to assist people but also to entertain.

\section{CONCLUSION}

This paper proposed the step-on interface (SOI) to operate a mobile robot in which a projector projects and displays a direction screen on a floor and a user specifies a button showing the selected movement by stepping or pointing. The HFAMRO-1 mobile robot has been developed to demonstrate the SOI's potential (HFAMRO: "human-friendly amusing" mobile robot). The SOI of HFAMRO- 1 consists of a projector and a range scanner on an omni-directional mobile platform. From operational comparison with other input interfaces, it is confirmed that we can direct the robot movement using our own foot. We had some students who does not specialize robotic systems try to operate HFAMRO1 with their shoes, and all trial students could specify the button to operate the robot satisfactorily and everyone mastered the SOI immediately.

In order to make the SOI and HFAMRO practical, it is necessary to solve various problems. The first problem is the detection of foot or shoes. Since the range scanner only detects the existence of object on a button, it reacts also to the wall and obstacles. We should examine the method to detect the shape of foot or shoes from the range data for the system to react it exclusively. Image processing using camera should also be considered. We would like to address the method to discriminate user's foot or shoes with various shapes and sizes, for example, by putting some mark on a tip of shoes to indicate a specified user. The second problem is downsizing and lightweight for robot mobility. Speeding up is necessary to interact with users, e.g. play tag, and downsizing and lightweight is required not to harm users. The third problem is multiple screens projected on the floor. We plan to develop the robot which projects screens all around it and examine creative usage and coupling control.

\section{ACKNOWLEDGMENTS}

Gratitude is expressed to laboratory members with selfsacrificing dedications, especially to Mr. Seigo Honda (now, Hitachi Ltd.) who contributed greatly on the setup movements of HFAMRO-1 and Mr. Yuichi Ito (now, master course student) who contributed on operational comparison with other input interfaces.

\section{REFERENCES}

[1] S. Honda, K. Akai, M. Lin, and T. Matsumaru, "Teleoperation of Human-Friendly Robot (41st report) -Examination of hardware constitution of HFAMRO-”, SICE-Chubu Shizuoka 2006, Dec 25 , 2006, Hamamatsu, Japan. Tokyo, Japan: SICE, 2006, 2 D1.

[2] K. Akai, S. Honda, M. Lin, and T. Matsumaru, "Teleoperation of Human-Friendly Robot (43rd report) -Examination of software constitution of HFAMRO-”, SICE-Chubu Shizuoka 2006, Dec 25, 2006, Hamamatsu, Japan. Tokyo, Japan: SICE, 2006, 2 D3.

[3] K. Akai, S. Honda, M. Lin, S. Suzuki, and T. Matsumaru, "Operation Method of Human-Friendly Robot (1st report) -Study on layout of step-on-interface-", In: Proc. of 51st Annual Conf. on the Institute of Systems, Control, and Information Engineers (SCI'07), May 16-18, 2007, Kyoto, Japan. Kyoto, Japan: ISCIE, 2007, 5T11.

[4] S. Honda, K. Akai, M. Lin, S. Suzuki, and T. Matsumaru, "Operation Method of Human-Friendly Robot (2nd report) -Study on behavior system using step-on-interface-”, In: Proc. of 51st Annual Conf. on the Institute of Systems, Control, and Information Engineers (SCI'07), May 16-18, 2007, Kyoto, Japan. Kyoto, Japan: ISCIE, 2007, 5T1-2.

[5] J. Raskin, The Humane Interface: New Directions for Designing Interactive Systems, Addison-Wesley Professional, Boston, MA, 2000.

[6] P. D. Wellner, "The DigitalDesk Calculator: Tactile Manipulation on a Desk Top Display", In: Proc. ACM SIGGRAPH Symp. on User Interface Software and Technology (UIST '91), Nov 11-13, 1991, Hilton Head, South Carolina, USA. New York, NY: ACM, 1991, pp. 27-33.

[7] H. Koike, Y. Sato, and Y. Kobayashi, "Integrating paper and digital information on enhanceddesk: A method for realtime finger tracking on an augmented desk system", ACM Transaction ComputerHuman Interaction (TOCHI), vol. 8, no. 4, pp. 307-322, 2001.

[8] J. Patten, H. Ishii, J. Hines, and G. Pangaro, "Sensetable: A Wireless Object Tracking Platform for Tangible User Interfaces", In: Proc. ACM SIGCHI Conf. on Human Factors in Computing Systems (CHI 2001), Mar 31-Apr 5, 2001, Seattle, Washington, USA. New York, NY: ACM, 2001, pp. 253-260.

[9] B. Piper, C. Ratti, and H.Ishii, "Illuminating Clay: A 3-D Tangible Interface for Landscape Analysis", In: Proc. ACM SIGCHI Conf. on Human Factors in Computing Systems (CHI 2002), Apr 20-25, 2002, Minneapolis, Minnesota, USA. New York, NY: ACM, 2002, pp. 355-362.

[10] J. Rekimoto, "SmartSkin: An Infrastructure for Freehand Manipulation on Interactive Surfaces", In: Proc. ACM SIGCHI Conf. on Human Factors in Computing Systems (CHI 2002), Apr 20-25, 2002, Minneapolis, Minnesota, USA. New York, NY: ACM, 2002, pp. 113-120.

[11] Y. Kakehi, M. Iida, T. Naemura, Y. Shirai, M. Matsushita, and T. Ohguro, "Lumisight Table: an interactive view-dependent tabletop display”, IEEE Computer Graphics and Applications, vol. 25, no. 1, pp. 48-53, 2005.

[12] M. Nikaido, M. Sugi, Y. Tamura, J. Ota, T. Arai, K. Kotani, K. Takamasu, A. Yamamoto, S. Shin, H. Suzuki, and Y. Sato, "Arrangement Planning for Multiple Self-Moving Trays in Human Supporting Production Cell `Attentive Workbench”, In: Proc. 2005 IEEE/RSJ Int'l Conf. on Intelligent Robots and Systems (IROS 
2005), Aug 2-6, 2005, Edmonton, Alberta, Canada. Piscataway, NJ: IEEE, 2005, pp. 3880-3885.

[13] N. Matsushita and J. Rekimoto, "HoloWall: Designing a Finger, Hand, Body, and Object Sensitive Wall", In: Proc. 10th annual ACM Symp. on User Interface Software and Technology 1997 (UIST'97), Oct 14-17, Banff, Alberta, Canada. New York, NY: ACM, 1997, pp. 209-210.

[14] Y. Nakanishi, Y. Sato, and H. Koike, "EnhancedDesk and EnhancedWall: Augmented Desk and Wall Interfaces with Real-Time Tracking of User's Motion", In: Proc. UbiComp 2002 Workshop on Collaborations with Interactive Walls and Tables, Sep 29 - Oct 1, Göteborg, Sweden. New York, NY: ACM, 2002, pp.27-30.

[15] S. Brave, H. Ishii, A. Dahley, "Tangible Interfaces for Remote Collaboration and Communication", In: Proc. 1998 ACM Conf. on Computer Supported Cooperative Work (CSCW '98), Nov 14-18, 1998, Seattle, Washington, USA. New York, NY: ACM, 1998, pp.169-178.

[16] J. D. Brederson, M. Ikits, C. R. Johnson, and C. D. Hansen, "The Visual Haptic Workbench", In: Proc. Fifth PHANToM Users Group Workshop '00 (PUG2000), Oct 28-30, 2000, Aspen, Colorado, USA. Boston, MA: MIT, 2000, pp.46-49.

[17] G. Pangaro, D. Maynes-Aminzade, and H. Ishii, "The Actuated Workbench: Computer-Controlled Actuation in Tabletop Tangible Interfaces", In: Proc. 15th annual ACM Symp. on User Interface Software and Technology (UIST 2002), Oct 27-30, 2002, Paris, France. New York, NY: ACM, 2002, pp. 181-190.

[18] H. Noma, S. Yoshida, Y. Yanagida, and N. Tetsutani, "The proactive desk: A new haptic display system for a digital desk using a 2DOF linear induction motor", Presence, vol. 13, no. 2, pp. 146-163, 2004.

[19] S. Hiura, K. Tojo, and S. Inokuchi, "3-D Tele-direction Interface using Video Projector", In: 30th Int'l Conf. on Computer Graphics and Interactive Techniques (ACM SIGGRAPH 2003) Sketches \& Applications, Jul 27-31, 2003, San Diego, California. New York, NY: ACM, 2003.

[20] T. Machino, Y. Nanjo, Y. Yanagihara, H. Kawata, S. Iwaki, and K. Shimokura, "Robot-augmented communication: a remotecollaboration system based on a shared field of view in real space", In: 2005 IEEE/RSJ Int'l Conf. on Intelligent Robots and Systems (IROS 2005), Aug 2-6, 2005, Edmonton, Alberta, Canada. Piscataway, NJ: IEEE, 2005, pp. 2203-2209.

[21] R. Raskar, P. Beardsley, J.V. Baar, Y. Wang, P. Dietz, J. Lee, D. Leigh, and T. Willwacher, "RFIG lamps: interacting with a selfdescribing world via photosensing wireless tags and projectors", ACM Transaction on Graphics (TOG), vol. 23, no. 3, pp. 406-415, 2003.

[22] M. Terashima, and S. Sakane, "A Human-Robot Interface Using an Extended Digital Desk”, In: Proc. 1999 IEEE Int'l Conf. on Robotics and Automation (ICRA'99), May 10-15, 1999, Detroit, Michigan, USA. Piscataway, NJ: IEEE, 1999, pp. 2874-2880.

[23] S. Sato, and S. Sakane, "A human-robot interface using an interactive hand pointer that projects a mark in the real work space", In: Proc. 2000 IEEE Int'l Conf. on Robotics and Automation (ICRA'00), Apr 24-28, 2000, San Francisco, California, USA. Piscataway, NJ: IEEE, 2000, pp. 589-595.

[24] M. Yamashita, and S. Sakane, "Adaptive Annotation Using a Human-Robot Interface System PARTNER", In: Proc. 2001 IEEE Int'l Conf. on Robotics and Automation (2001 ICRA), May 21-26, 2001, Seoul, Korea. Piscataway, NJ: IEEE, 2001, pp. 2661-2667.

[25] Y. Wakita, S. Hirai, T. Suehiro, T. Hori, and K. Fujiwara, "Information Sharing via Projection Function for Coexistence of Robot and Human", Autonomous Robots, vol. 10, no. 3, pp. 267-277, 2001.
[26] T. Matsumaru, "Mobile Robot with Preliminary-announcement and Display Function of Following Motion using Projection Equipment", In: 15th IEEE Int. Symp. on Robot and Human Interactive Communication (IEEE RO-MAN 06), Sep 6-8, 2006, Hatfield, United Kingdom. Piscataway, NJ: IEEE, 2006, pp. 443-450.

[27] T. Matsumaru: "Operating Device", Patent Abstracts of Japan, 2008-006551, Jan 17 2008. [On-Line] Available www.ipdl.inpit.go.jp/homepg_e.ipdl [Accessed Nov. 15, 2008].

[28] A. Holzinger, "Finger Instead of Mouse: Touch Screens as a means of enhancing Universal Access", In: N. Carbonell, and C. Stephanidis (Eds), "Universal Access, Theoretical Perspectives, Practice, and Experience", Springer, Berlin, Germany, 2003, pp. 387-397.

[29] S. Kanda, M. Yuichi, N. Sawasaki, and T. Asada, "Development of the Service Robot "enon", Journal of the Robotics Society of Japan, vol. 24, no.3, pp. 288-291, 2006.

[30] H. Asoh, I. Hara, and T. Matsui, "Dynamic Structured Multi-Agent Architecture for Controlling Office-Conversant Mobile Robot", In: Proc. 1998 IEEE Int'l Conf. on Robotics and Automation (ICRA'98), May 16-20, 1998, Leuven, Belgium. Piscataway, NJ: IEEE, 1998, pp. 1552-1557.

[31] K. Nakadai, D. Matsuura, H.G. Okuno, and H. Kitano, "Applying scattering theory to robot audition system: Robust sound source localization and extraction", In: Proc. IEEE/RSJ Int'l Conf. on Intelligent Robots and Systems (IROS 2003), Oct 27-31, 2003, Las Vegas, USA. Piscataway, NJ: IEEE, 2003, pp. 1157-1162.

[32] T. Shibata, Y. Matsumoto, T. Kuwahara, M. Inaba, and H.Inoue, "Hyper Scooter: A mobile Robot sharing visual information with a human", In: Proc. 1995 IEEE Int'l Conf. on Robotics and Automation (ICRA 1995), May 21-28, 1995, Nagoya, Japan. Piscataway, NJ: IEEE, 1995, pp. 1074-1079.

[33] S. Esaki, Y. Ebisawa, A. Sugioka, and M. Konishi, "Quick menu selection using eye blink for eye-slaved nonverbal communicator with video-based eye-gaze detection", In: Proc. 19th Annual Int'l Conf. of the IEEE Engineering in Medicine and Biology Society (IEEE EMBS 1997), Oct 30 - Nov 2, 1997, Chicago, IL, USA. Piscataway, NJ: IEEE, 1997, pp. 2322-2325.

[34] Center for Art and Media in Karlsruhe, "Small Fish", Center for Art and Media in Karlsruhe, 2002. [On-line] Available: hosting.zkm.de/wmuench/small_fish [Accessed Nov 15, 2008].

[35] Solidray Co. Ltd., "Taptalk", Solidray Co. Ltd., 2008. [On-line] Available: www.taptalk.jp [Accessed Nov. 15, 2008].

[36] H.E. Korth, "Method and device for optical input of commands or data", EP patent, 0554492, Feb. 07 1992. [On-Line] Available www.freepatentsonline.com/5767842.html [Accessed Nov 15, 2008].

[37] Hokuyo Automatic Co. Ltd., "Photo sensor", Hokuyo Automatic Co. Ltd., 2008. [On-line] Available: www.hokuyo-aut.jp/02sensor/ [Accessed Nov 15, 2008].

[38] M. Lin, H. Yamamori, S. Honda, T. Matsumaru, "Teleoperation of Human-Friendly Robot (42nd report) -Examination of foot position sensing with a URG sensor-", SICE-Chubu Shizuoka 2006, Dec 25, 2006, Hamamatsu, Japan. Tokyo, Japan: SICE, 2006, 2 D2.

[39] DHL-AIST, "Japanese Body Dimension Data, 1997-98", AIST, 2005. [On-Line] Available www.dh.aist.go.jp/database/bodydimeDB/ [Accessed Nov 15, 2008].

[40] Wikimedia Foundation, Inc., "Key arrangement", Wikimedia Foundation, Inc., 2008. [On-line] Available: ja.wikipedia.org/wiki/ [Accessed Nov 15, 2008].

[41] Julius development team, "Julius for SAPI", Julius development team, 2008. [On-line] Available: julius.sourceforge.jp/sapi/ [Accessed Nov 15, 2008].

This is an open access article licensed under the terms of the Creative Commons Attribution Non-Commercial License (http://creativecommons.org/licenses/by-nc/3.0/) which permits unrestricted, non-commercial use, distribution and reproduction in any medium, provided the work is properly cited. 